\title{
Investigation and management of a large community mumps outbreak among young adults in Toronto, Canada, January 2017-February 2018
}

\author{
V Dubey ${ }^{1,2 \star}$, O Ozaldin ${ }^{1}$, L Shulman ${ }^{1}$, R Stuart ${ }^{1}$, J Maclachlan ${ }^{1}$, L Bromley ${ }^{1}$, A Summers ${ }^{2}$
}

\section{Abstract}

Background: In 2017, a mumps outbreak was identified in a cohort of 18-34 year olds in Toronto, Canada.

Objective: To describe a large community mumps outbreak in an urban centre from January 2017 to February 2018 among young adults.

Methods: A broad range of interventions were implemented in an attempt to reach the target audience; including case and contact management, vaccination clinics at schools and clinicians' offices, school exclusions, bar inspections, traditional communication strategies (including health care provider updates and posters) and newer communication strategies (including three sequential social media campaigns).

Results: A total of 143 cases of mumps were identified. Although cases' ages ranged from three to 72 years, most (76\%) were 18-34 year olds, many of whom had frequented bars and local food establishments in downtown Toronto. Eighty-four percent $(n=120)$ of the cases were community-acquired. Only $16 \%(n=23)$ of the cases reported exposures in schools and post-secondary school institutions. Of those, $39 \%(n=56)$ of cases had an unknown vaccination history; $34 \%(n=49)$ were either not vaccinated or partially vaccinated with one dose of measles-mumps-rubella vaccine; and $27 \%(n=38)$ had received the recommended two doses of mumps vaccine. Determining vaccination status was a challenge, in part due to the lack of a registry. Vaccination was recommended when subjects were known to have had fewer than two doses of vaccine or had an unknown vaccination status. A social media campaign, emphasizing the risk of social activities if not protected from the mumps, yielded over 500,000 impressions from Facebook and Twitter messages and ads and an impressive engagement rate of between $1 \%$ and $10 \%$.

Conclusion: This was the largest mumps outbreak in Toronto in over 20 years. Among young adults, ongoing social media and traditional communication campaigns can contribute to the control of community mumps outbreaks. Encouraging vaccine uptake is desirable, but without a vaccine registry it is difficult to assess vaccination coverage among adults. Susceptible cohorts of young adults who were not adequately vaccinated pose a risk for future outbreaks. Given that almost $30 \%$ of the mumps cases were fully vaccinated with two doses of mumps-containing vaccine, even two doses may not provide complete protection.

\section{Affiliations}

${ }^{1}$ Toronto Public Health, Toronto, ON

${ }^{2}$ Dalla Lana School of Public Health, University of Toronto, Toronto, ON

\section{*Correspondence:}

vinita.dubey@toronto.ca

Suggested citation: Dubey V, Ozaldin O, Shulman L, Stuart R, Maclachlan J, Bromley L, Summers A. Investigation and management of a large community mumps outbreak among young adults in Toronto, Canada,

January 2017-February 2018. Can Commun Dis Rep 2018;44(12):309-16. https://doi.org/10.14745/ccdr. v44i12a01

Keywords: mumps, outbreak, mumps vaccine, social media, vaccine coverage, vaccine registry, young adults, MMR, Toronto 


\section{Introduction}

From January 2017 to February 2018, Toronto experienced the largest mumps outbreak in the city in over 20 years with 143 cases. Toronto is Canada's largest urban centre with a population of 2.7 million. Toronto has an average of five cases of mumps per year; largely travel-related. The last large outbreak in Toronto was in 2009, with 33 cases.

Mumps is a viral infection caused by a paramyxovirus, which can lead to symptoms of fever, malaise, headache, myalgia and parotitis. Orchitis is a common complication in postpubertal males. Although a third of cases have only mild symptoms, complications include meningitis, pancreatitis, myocarditis and deafness. Symptoms are often more severe in adults than children. The incubation period is $12-25$ days, and communicability through droplet and direct contact with saliva or respiratory droplets occurs from seven days before to five days after onset of symptoms. Contagiousness is similar to that of influenza (1-3).

Adults born before 1970 are generally presumed to have acquired natural immunity to mumps. In Ontario, a single dose of measles-mumps-rubella (MMR) vaccine was provided from 1975 to 1996. In 1996, a second dose of MMR vaccine was added to the schedule and a single dose of monovalent measles vaccine was offered to all students $4-18$ years of age (born in 1978 to 1992) $(4,5)$. Coverage rates for two doses of mumps-containing vaccines among school-aged children has consistently been about $90 \%$ for the past ten years in Toronto schools $(6,7)$.

This vaccination plan has left a cohort of individuals born after 1970 and before 1992 who received only one dose of mumps-containing vaccine. The National Advisory Committee of Immunization (NACl) has recommended that during a mumps outbreak, this age cohort receives a dose of mumps-containing vaccine; however, this cohort is notoriously difficult to reach (8).

Vaccine registries are important tools to document and improve coverage. When vaccine preventable disease outbreaks occur, a registry can confirm previous vaccinations and readily assess susceptible individuals in the defined population who require vaccination.

The objective of this article is to describe this recent large community mumps outbreak in Toronto and novel approaches for communication and outbreak control using social media and posters.

\section{Outbreak detection}

The outbreak began in January 2017 when two unvaccinated siblings (18 and 20 years of age) were reported to Toronto Public Health with laboratory-confirmed mumps infections. Both had symptoms of fever, fatigue and parotitis. It was determined that the infection was likely acquired during a house party in Guelph, Ontario (small city approximately 100 kilometres south west of Toronto) in mid-January. Cases were also detected across Ontario related to this house party exposure. Additional cases of mumps were then detected in young adults with links to downtown Toronto bars and food establishments that had no identified connections to the Guelph house party, travel or other cases. An outbreak of mumps for the City of Toronto was declared on January 30, 2017.

\section{Outbreak response}

\section{Case definitions and investigations}

The outbreak case definitions are summarized in Appendix 1. In Ontario all laboratory specimens for mumps were reported directly to the local public health unit for follow up as per the Ontario Public Health Standards Infectious Diseases Protocol (9). Public health staff then interviewed all cases, utilizing an Integrated Public Health Information System (iPHIS) case investigation tool that was customized for this specific outbreak. Clients were asked to provide information on their vaccination history, symptoms, occupation, attendance at medical and school settings, medical and social risk factors and potential acquisition and transmission exposure sites. Early in the outbreak, it became clear that clients were not forthcoming with their answers to all of the questions, especially those questions relating to details of contacts and possible exposure sites.

The Public Health Ontario Laboratory forwarded specimens to the National Microbiology Laboratory (NML) for genotyping. Due to delays in receiving results, genotyping was not included in the case definitions.

Descriptive analyses to assess the demographics, geography, vaccination status, genotype and symptoms associated with cases were performed. Social networking analysis was contemplated early in the outbreak, however since cases were not forthcoming with all of their exposures and social networks, there was not enough information to pursue this analysis.

\section{Case and contact management}

Conventional case management of mumps was undertaken $(3,9)$. Cases were asked to self-isolate and were excluded from work, school, social gatherings and health care facilities during the period of communicability (five days after onset of symptoms). Interviews were completed with cases in order to identify potential sites of acquisition and transmission during the incubation period and period of communicability. Contact management, as used in the outbreak, is summarized in Appendix 2.

\section{Health care provider updates}

The majority of vaccinations in Ontario are provided by primary care clinicians. Numerous messages were sent to vaccine providers to update them on the status of the outbreak, to provide instructions on how to diagnose and test for mumps infection and 
to encourage them to vaccinate their 18 to 35 year old patients. Because mumps-containing vaccines in Toronto are ordered by providers and shipped from the Ontario Government Pharmacy and Medical Supply Service, the Panorama vaccine inventory database was used to determine mumps-containing vaccines that were ordered and shipped from March to August in 2017 compared with the same time period in 2018 (post-outbreak).

\section{Mandated exclusion of susceptible student contacts from school}

Ontario's Immunization of School Pupils Act requires that all students are either vaccinated against certain diseases or have submitted a medical or philosophical/religious exemption (10). In the context of an outbreak, public health officials may exclude students who are not up-to-date with their vaccinations or do not have evidence of immunity. In schools where a case was reported, attention was given to update vaccine records and to vaccinate those who were not up-to-date with two doses of mumps-containing vaccines. In one high school where there were two cases with possible transmission in the school setting, students who were not up-to-date with their vaccinations or who were non-immune to mumps were excluded from school until they could provide proof of vaccination. Vaccine clinics were held at schools to update vaccination records and to quickly vaccinate staff and students. Further transmission in elementary and high schools did not occur.

\section{Bar inspections}

In the initial phase of the outbreak, bars that had been visited by confirmed cases during their period of communicability were inspected. The inspections focused on potential infection prevention and control lapses that might have explained the transmission, such as inadequate dish and glass cleaning and disinfection. A letter and fact sheet on mumps were developed and given to bar owners.

\section{Communication strategy}

A communication strategy was developed to target young adults who commonly attended bars in the west downtown core of Toronto. The key messages focused on educating the target audience about mumps infection and transmission, and promoting vaccination. Over 70 media interviews were conducted via multiple media and news outlets. Letters and posters were created and distributed to various audiences in an attempt to reach the target young adults (Figure 1). Community centres were accessed through internal city listings and gyms were identified through listings available online. All post-secondary institutions in Toronto were identified and sent materials in August 2017 in advance of "frosh week" and the start of classes.

Since many cases had listed downtown bars and restaurants as possible exposure settings early in the outbreak, over 4,000 letters were mailed to downtown bars and restaurants. Many staff members in these bars were identified as cases, so posters aimed
Figure 1: Sample poster and social media image used for Toronto mumps outbreak, 2017-2018

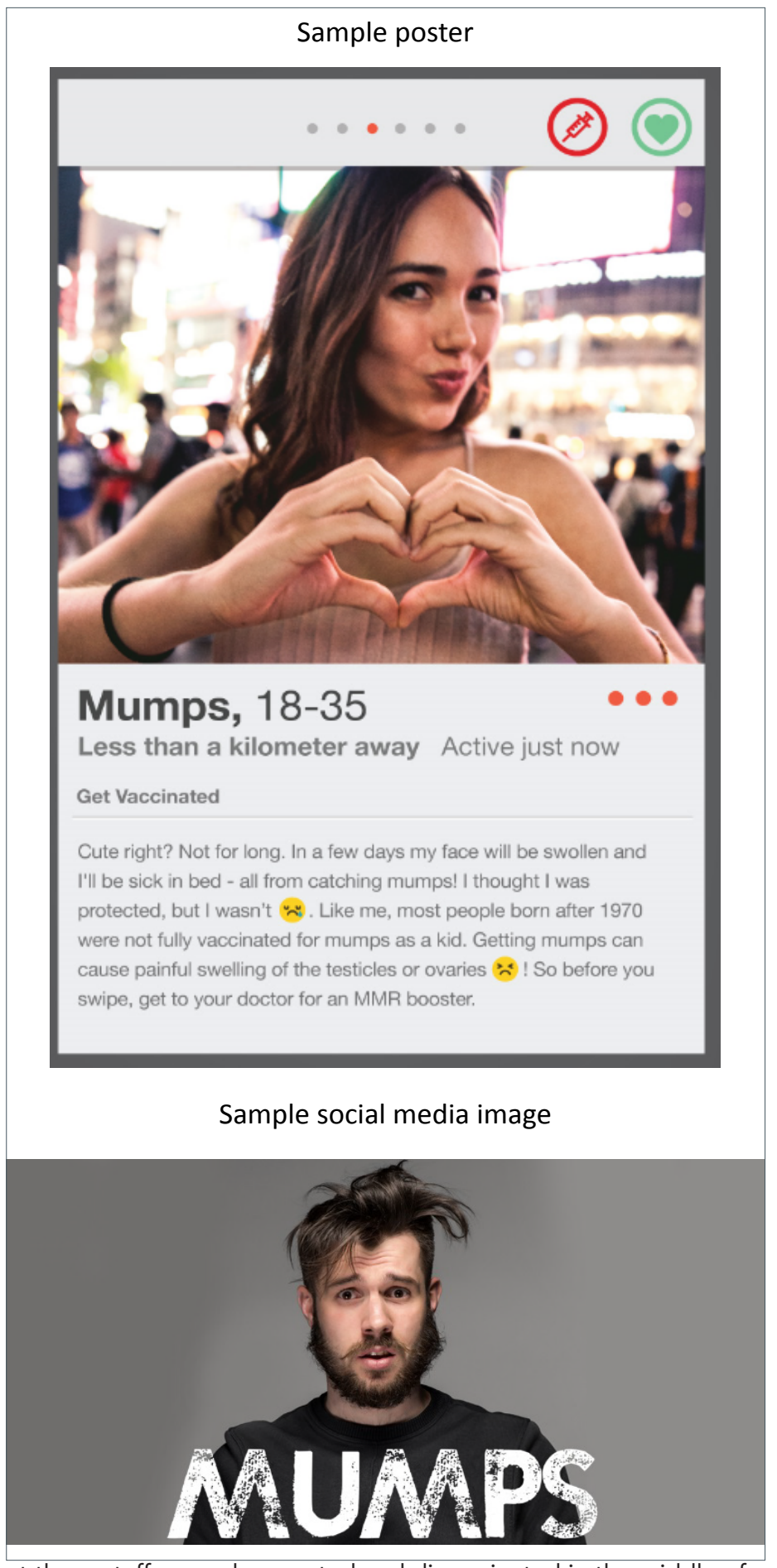

at these staff were also created and disseminated in the middle of the outbreak.

An outbreak webpage was created and updated regularly with new case counts and prevention messages.

\section{Social media strategy}

Three social media campaigns were launched throughout the outbreak on Facebook and Twitter. In the first wave of the 
outbreak, a social media campaign ran from February to April 2017, targeting socially-active young people in Toronto's downtown west end. The goal was to raise awareness that mumps was circulating in Toronto and to encourage the target audience to check vaccination records or speak with their doctor to make sure that vaccinations were up to date. Creative images were designed to reflect the style, attitudes and online behaviours of the target audience (Figure 1). Sample social media messages used during the Toronto mumps outbreak included the following:

- $\quad$ Spread love, not mumps. Don't share drinks, utensils, food or water bottles

- Your style is up to date, but are your vaccines? Make sure you are protected from mumps

- Mumps is more than a funny word-it's on the rise in Toronto

- Catch feelings this summer, not mumps. Talk to your doctor about the MMR

The second social media campaign ran from July to September 2017, with an updated creative design and a stronger call to action. As it became clear that the outbreak was not ending and increasing herd immunity was essential, "learn more" messages were repositioned to "get vaccinated". The images and messages were reworked to relate to the summer events that might lead to possible increased transmission.

Following another wave of cases in the fall, a campaign in December focused on images and messages updated with winter and holiday images. The main message was to get vaccinated.

\section{Results}

\section{Description of the outbreak}

A total of 143 cases of mumps were identified from January 1, 2017 to February 26, 2018. The outbreak had an initial peak in early March 2017, and by June 2017 the cases has declined substantially (Figure 2). A second peak began in late August and lasted into the

Figure 2: Epidemic curve of confirmed mumps cases in Toronto by week, January 1, 2017 to February 26, 2018

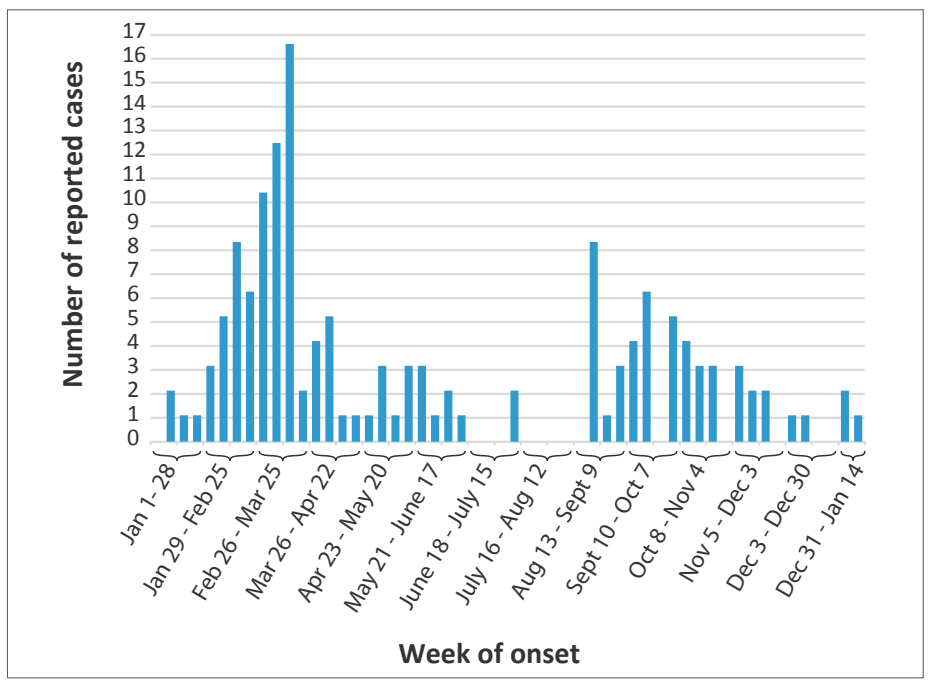

fall, and then declined throughout the rest of 2017. The mumps outbreak was declared over on February 26, 2018; 50 days (two incubation periods) after the onset in the last case.

Seventy-six percent of cases were between the ages of 18 to 34 years. The mean age of cases in the outbreak was 28 years old (range of 3-72 years old). The cases were fairly evenly distributed between genders (55\% male). Most ( $84 \%$ ) of the cases were community-acquired and only $16 \%$ of the cases were either a staff or a student at an elementary, high school or post-secondary institution; sustained transmission in these settings did not occur (Table 1).

Table 1: Descriptive summary of Toronto mumps cases, January 1, 2017 to February 26, 2018

\begin{tabular}{|c|c|c|}
\hline \multicolumn{3}{|c|}{ Reported cases } \\
\hline $\begin{array}{c}\text { Descriptive } \\
\text { characteristics }\end{array}$ & $\mathrm{n}$ & $\%$ \\
\hline Total number of cases & 143 & 100 \\
\hline \multicolumn{3}{|l|}{ Age $^{a}$ (years) } \\
\hline $0-5$ & 1 & 1 \\
\hline $6-11$ & 0 & 0 \\
\hline $12-17$ & 9 & 6 \\
\hline $18-25$ & 44 & 31 \\
\hline $26-34$ & 65 & 45 \\
\hline $35-49$ & 21 & 15 \\
\hline $50-64$ & 2 & 1 \\
\hline $65+$ & 1 & 1 \\
\hline \multicolumn{3}{|l|}{ Gender } \\
\hline Male & 79 & 55 \\
\hline Female & 64 & 45 \\
\hline \multicolumn{3}{|l|}{ School exposures ${ }^{b}$} \\
\hline Yes & 11 & 8 \\
\hline No & 132 & 92 \\
\hline \multicolumn{3}{|l|}{ Bar exposures ${ }^{b}$} \\
\hline Yes & 70 & 49 \\
\hline No & 73 & 51 \\
\hline \multicolumn{3}{|c|}{ Post-secondary school exposures ${ }^{b}$} \\
\hline Yes & 11 & 8 \\
\hline No & 132 & 92 \\
\hline \multicolumn{3}{|l|}{ Vaccination status } \\
\hline Vaccinated & 38 & 27 \\
\hline Not vaccinated & 16 & 11 \\
\hline Partially vaccinated & 33 & 23 \\
\hline Unknown & 56 & 39 \\
\hline
\end{tabular}


Parotitis was the most common symptom, reported by $97 \%(n=139)$ of cases. Serious complications were rare among cases: only two of the 143 cases visited the emergency room for their symptoms, and only one of those cases was admitted. Orchitis was reported by $23 \%(n=18)$ of male cases.

Most cases (73\%) were either not vaccinated (11\%), partially vaccinated with one dose of MMR vaccine $(23 \%)$ or had an unknown vaccination history (39\%). Only $27 \%$ had known vaccination with two doses of MMR vaccine. Five cases (3\%) born before 1970, who were presumed to be immune by age, also developed the mumps.

Most of the cases in the outbreak were locally-acquired (93\%). Of the 139 cases that were tested for genotype, the majority $(n=115)$ were genotype $G$. Other genotypes identified included one genotype $\mathrm{C}$ and one genotype $\mathrm{K}$, both travel-related. The travel-related cases were included in this outbreak because they were in Toronto for at least part of their acquisition period and the genotyping information was not included in the case definitions. The remainder $(n=22)$ were indeterminate.

Initially, most new cases were not clearly linked to each other or to common institutions; however, on epidemiologic assessment, cases were found to be geographically located in west downtown Toronto and common exposures at dozens of west downtown Toronto bars and restaurants were noted, either from a patron or a staff member at these establishments. As the outbreak progressed, the majority of cases were no longer reporting only bar exposures or west downtown Toronto exposures, and wide spread community transmission across the city was evident.

\section{Mumps-containing vaccine orders by primary care providers}

During the period from March to August 2017, a total of 78,680 doses of mumps-containing vaccine (MMR) were shipped by the Ontario Government Pharmacy and Medical Supply Service in orders from Toronto health care providers, which was an average of 13,113 doses per month. In the same period in 2018 (March to August), only 66,509 mumps-containing vaccines were shipped, with an average of 11,085 doses per month. This represents an increase of 12,000 doses shipped during the peak outbreak period in 2017, compared with a similar period the following year.

\section{Performance of social media campaigns}

The outcome of the social media messages exceeded expectations. For the first campaign from February to April, 2017 during the peak of the first wave of the outbreak, there were over 360,000 impressions and over 14,000 engagements from the Facebook and Twitter messages and ads. The engagement rate on Twitter reached $10 \%$, compared with the Toronto Public Health corporate account, which nearly averaged 1\%. For the 2017 summer campaign, the engagement rate on Facebook and Twitter was still high, at $1 \%$, and the accounts achieved an additional 50,000 impressions. The third campaign in December again maintained a high engagement rate, at $2 \%$, with almost 120,000 impressions.

The reception to the campaign was evaluated by monitoring the comments and reactions to the campaign messages. Overall positive responses (likes, loves and laughs) far outnumbered the negative. People liked the humorous approach and noted the importance of vaccination. As expected, anti-vaccination comments were also present.

The mumps outbreak investigation webpage had a substantial increase in web traffic, from 161 visits in January 2017 to 13,698 visits from February to April 2017 at the height of the outbreak. Web hits increased when there was high media coverage, retweets by influential people and Facebook ads.

\section{Discussion}

This community-based outbreak predominately made up of young adults aged 18-34 years began in a distinct geographic area in west downtown Toronto bars and restaurants, and spread throughout the city.

Although some of the young adults were part of the cohort born after 1970 and before 1992 that had only one mumps-containing vaccine as a child, $50 \%$ of cases had an unknown vaccination status or were not vaccinated. Five people (3\%) born before 1970, who were presumed to be immune by age, also developed the mumps. Without a vaccine registry, it is difficult to determine how many of those who had unknown vaccination status were actually vaccinated. A registry would also enable calculations of time since last vaccination which may be an important indicator of mumps vaccine-derived immunity in an outbreak setting (11).

This outbreak presented unique challenges in contact tracing and public health messaging, especially since the outbreak did not begin in an institution or well-defined group of individuals. There were difficulties reaching the clients through traditional phone calls and letters. Many were reluctant to provide contact information for their symptomatic close contacts (friends, coworkers or casual sexual partners) so it was left to the cases to notify their contacts. Some cases worked at a food establishment and were reluctant to provide their work information because they were concerned about negative publicity for the food establishment and the risk of termination.

Most young adults prefer to communicate and receive information through texting and social media rather than through more conventional methods such as newspapers and letters. Platforms such as Twitter and Facebook were identified as the ideal channels to quickly and efficiently engage the target audience. The challenge was to make the public health message relevant, engaging and urgent to a younger demographic. In this outbreak, we found that many young adults assumed they were fully vaccinated so the vaccination message did not seem relevant to 
them. They did not feel vulnerable to illness nor did they perceive an urgency for vaccination. To address this, a social media strategy focused on the 'hipster' target audience, highlighting the social consequences of falling ill, such as missing social events and feeling left out. The response was generally positive, similar to that reported in other outbreaks (12).

Many cases and contacts had a difficult time finding their vaccination records (39\% of cases). Encouraging vaccination rather than serologic testing of immunity in someone with unknown records became an important message to health care providers. Without a registry, it was difficult to say how many people were vaccinated in response to the outbreak; however, a proxy measure, vaccines distributed to providers, showed an increase in orders for mumps-containing vaccines during the height of the outbreak.

Other large mumps outbreaks have been reported in North America in recent years and the majority of these outbreaks have occurred in schools, colleges or sports teams, and many have been reported in populations assumed to be fully-vaccinated $(13,14)$. Recently, the Advisory Committee on Immunization Practices in the United States has recommended a third dose of mumps-containing vaccine in an outbreak setting where there is already high two-dose coverage among cases (11). In the Toronto outbreak, almost a third of cases $(27 \%)$ occurred in fully-vaccinated adults.

It is often difficult to determine why an outbreak ends. This was a thirteen-month community outbreak in a large urban centre. Sustained transmission in the schools did not occur. Public health messaging to modify social behaviours, such as sharing utensils while in a bar and restaurant setting, may also have been important. Increased vaccination likely played a role in ending this outbreak. Although an excess of 12,000 doses of vaccine were given in a six-month period during the height of the outbreak compared with the subsequent year, it is difficult to determine how large the susceptible cohort of young adults remains in Toronto without a registry.

\section{Limitations}

Underreporting of cases is likely for a number of reasons: improper, incomplete or no testing from clinicians; mild or asymptomatic cases who were less likely to seek medical attention; and some cases who were reluctant to report symptomatic contacts. Immunization status was difficult to verify as cases and contacts often did not have records available.

\section{Conclusion}

Among susceptible cohorts of young adults, ongoing social media and traditional communication campaigns can contribute to the control of community mumps outbreaks. Encouraging vaccine uptake is desirable, but without a vaccine registry it is difficult to assess vaccination coverage among adults. Susceptible cohorts of young adults who were not adequately vaccinated because of historic vaccination policies pose a risk for future outbreaks. Additionally, given that almost $30 \%$ of the mumps cases were fully vaccinated with two doses of mumps-containing vaccine, even two doses may not provide complete protection.

\section{Authors' statement}

All of the authors had access to the data, contributed to the preparation and revision of the manuscript and approved the final version.

\section{Conflict of interest}

None.

\section{Acknowledgments}

The authors would like to thank all the staff members from Toronto Public Health who were part of the outbreak management team, the vaccine preventable diseases team and those who participated in the social media and communication campaigns, as well as our provincial public health colleagues.

\section{References}

1. Heymann DL, editor. Control of Communicable Diseases Manual. 20th edition. Washington (DC): American Association of Public Health; 2014.

2. Centers for Disease Control and Prevention. Epidemiology and Prevention of Vaccine-Preventable Diseases. Hamborsky J, Kroger A, Wolfe S, editors. 13th ed. Washington (DC): Public Health Foundation; 2015. 12 p. www.cdc.gov/ vaccines/pubs/pinkbook/index.html

3. Public Health Agency of Canada. Guidelines for the prevention and control of mumps outbreaks in Canada. Can Commun Dis Rep 2010;36 Suppl:1-46. DOI

4. Watson-Creed G, Saunders A, Scott J, Lowe L, Pettipas J, Hatchette TF. Two successive outbreaks of mumps in Nova Scotia among vaccinated adolescents and young adults. CMAJ 2006 Aug;175(5):483-8. DOI PubMed

5. Deeks SL, Lim GH, Simpson MA, Gagné L, Gubbay J, Kristjanson E, Fung C, Crowcroft NS. An assessment of mumps vaccine effectiveness by dose during an outbreak in Canada. CMAJ 2011 Jun;183(9):1014-20. DOl PubMed

6. Toronto Public Health. Childhood Immunization Coverage in Toronto - Report from Medical Officer of Health, January 26, 2009. www.toronto.ca/legdocs/mmis/2009/hl/bgrd/ backgroundfile-18659.pdf

7. Ontario Agency for Health Protection and Promotion (Public Health Ontario). Immunization Coverage Report for School Pupils in Ontario: 2016-17 School Year. Toronto (ON): Queen's Printer for Ontario; 2018. www. publichealthontario. ca/en/eRepository/immunization-coverage-report-2016-17. pdf

8. National Advisory Committee on Immunization. Mumps Vaccine: Canadian Immunization Guide. Ottawa (ON): PHAC. 
www.canada.ca/en/public-health/services/publications/ healthy-living/canadian-immunization-guide-part-

4-active-vaccines/page-14-mumps-vaccine.html

9. Ontario Public Health Standards, Infectious Diseases Protocol, Appendix A: Disease-Specific Chapters - Mumps. Revised January 2014. www.health.gov.on.ca/en/pro/ programs/publichealth/oph_standards/docs/mumps_ chapter.pdf

10. Ontario Provincial Government, Immunization of School Pupils Act. R.S.O. 1990, Chapter I.1. September 2017. www. ontario.ca/laws/statute/90i01

11. Marin M, Marlow M, Moore KL, Patel M. Recommendation of the Advisory Committee on Immunization Practices for Use of a Third Dose of Mumps Virus-Containing Vaccine in Persons at Increased Risk for Mumps During an Outbreak. MMWR Morb Mortal Wkly Rep 2018 Jan;67(1):33-8. DOI PubMed
12. Ross C, Shaw S, Marshall S, Stephen S, Bailey K, Cole R, Wylie J, Bullard J, Van Caeseele P, Reimer J, Plourde P. Impact of a social media campaign targeting men who have sex with men during an outbreak of syphilis in Winnipeg, Canada. Can Commun Dis Rep 2016 Feb;42(2):45-9. DOI PubMed

13. Bonwitt J, Kawakami V, Wharton A, Burke RM, Murthy $N$ Lee A, Dell B, Kay M, Duchin J, Hickman C, McNall RJ, Rota PA, Patel M, Lindquist S, DeBolt C, Routh J. Notes from the Field: Absence of Asymptomatic Mumps Virus Shedding Among Vaccinated College Students During a Mumps Outbreak - Washington, February-June 2017. MMWR Morb Mortal Wkly Rep 2017 Dec;66(47):1307-8. DOI PubMed

14. Albertson JP, Clegg WJ, Reid HD, Arbise BS, Pryde J, Vaid A, Thompson-Brown R, Echols F. Mumps Outbreak at a University and Recommendation for a Third Dose of Measles-Mumps-Rubella Vaccine - Illinois, 2015-2016. MMWR Morb Mortal Wkly Rep 2016 Jul;65(29):731-4. DOI PubMed

\section{Appendix 1}

\section{Case definitions use in Toronto mumps outbreak, January 1, 2017 to February 16, 2018}

\section{Confirmed}

A resident of or visitor to Toronto with the following:

1. Laboratory confirmation of infection with a specimen collection date on or after January 1, 2017 with clinical signs and symptoms compatible with mumps infection with symptom onset on or after January 1, 2017

OR

2. Clinically compatible signs and symptoms with mumps infection with onset on or after January 1, 2017 in a person with an epidemiologic link to a laboratory-confirmed outbreak case AND

3. Not linked to a travel-related exposure

\section{Probable}

A resident or visitor to Toronto with the following:

1. Clinical sign and symptoms compatible with mumps infection with symptom onset on or after January 1, 2017

AND

2. A link to a known outbreak related exposure site (absence of an epidemiologic link to a laboratory-confirmed case)

AND

3. Absence of laboratory testing or laboratory confirmation (e.g. laboratory results are pending and or it is outside the window of laboratory testing sensitivity)

AND

4. Not linked to a travel-related exposure 


\section{Appendix 2}

\section{Contact management for Toronto mumps outbreak, January 1, 2017 to February 16, 2018}

\section{Determination of contact}

Contacts were defined by fulfillment of at least one of the following criteria during the infectious period (i.e., seven days before to five days after symptom onset):

1. Household contacts of a case

2. Persons who share sleeping arrangements with the case, including shared rooms (e.g., dormitories)

3. Direct contact with the oral/nasal secretions of a case (e.g., face-to-face contact, sharing cigarettes/drinking glasses/food/cosmetics like lip gloss, kissing on the mouth)

4. Children and staff in child care and school facilities

5. Health care workers with unprotected face-to-face interaction within one metre of an infectious mumps case

6. Individuals who share the same indoor air space with the case for more than one hour (e.g., during small social gatherings, such as birthday parties and sports teams)

\section{Management of contacts}

For contacts who met the above criteria, the following were done

1. advise contacts of possible exposure to mumps and educate about disease transmission

2. determine the immunization status of all contacts; encouraging vaccination of unimmunized or under-immunized individuals

3. note any symptoms, onset and severity; and

4. consider all symptomatic contacts as probable cases and perform confirmatory testing

\section{Notification of contacts}

Contact notification was done by public health in certain situations such as health care institutions or schools and if resources permitted; however, with a large number of cases in the outbreak it was not feasible. Contact notification by the case was used. Cases informed their contacts, including workplaces, usually electronically or by phone, about their potential exposure and provided a letter from TPH and fact sheet.

\section{Susceptible contacts}

Those who may require exclusion from a health care or school setting include:

1. Those born in Canada in 1970 or later who did not receive two doses of mumps-containing vaccine (at least four weeks apart) on or after their first birthday

2. Those without past history of laboratory confirmed mumps; and

3. Those without documented immunity to mumps 